\title{
Role of vitamin D supplementation in burka clad pregnant women and its correlation with feto- maternal outcome-----a study in 450 bedded maternity hospital of walled city of Delhi
}

\begin{abstract}
Objective: Estimation of vitamin D level at term pregnancy and role of vitamin D supplementation on feto-maternal outcome in burka clad pregnant women.

Methods: 200 burka clad pregnant women of 12-16 weeks gestationattending antenatal clinic in Kasturba Hospital, Delhi, were selected. They were randomly assigned into two groups that is study group and control group. Study group comprised of 100 pregnant burka clad women who received vitamin D supplementation in form of oral cholecalciferol sachet $60,000 \mathrm{IU}$ in 5 th, 6th and 7th month of gestation along with routine iron, FA and calcium supplementation. Control group comprised of 100 pregnant burka clad women who only received routine iron, FA and calcium supplementation. At term pregnancy vitamin D levels were estimated in both groups by xpress ELISA kit and role of vitamin D supplementation in burka clad pregnant women and feto-maternal outcome of the two groups were compared.

Results: Vitamin D supplementation during pregnancy was associated with increased circulating $25(\mathrm{OH})$ vitamin D levels at term. Vitamin D supplementation reduces the incidence of pre-eclampsia and low birth weight neonates. Incidence of GDM, preterm birth, cesarean section, NICU admission, tetany in newborn was not influenced by vitamin D supplementation.
\end{abstract}

Conclusion: At present, vitamin D supplementation is not a part of antenatal care programs in India. For recommendations concerning routine vitamin D supplementation during pregnancy; further clinical trials are required with larger sample size.

Keywords: vitamin D, pre-eclampsia, gestational diabetes mellitus, low birth weight, nicu admission, preterm birth
Volume 9 Issue 2 - 2018

Sangita Nangia Ajmani,' Manisha Uddey, ${ }^{2}$ Leela Pant, 3 Poonam Chauhan, 'Ajmani AK ${ }^{4}$ 'Obstetrics and Gynecology, Kasturba hospital, India

${ }^{2}$ Postgraduate student, Kasturba hospital, India

${ }^{3}$ Pathology, Kasturba hospital, India

${ }^{4}$ Medicine, Endocrinology, BL Kapur hospital, India

Correspondence: Dr. Sangita Nangia Ajmani, Obstetrics and Gynecology, HOD (Obstetrics and Gynecology), Officer Incharge HRRC, Kasturba hospital, India, Tel 9650403404, Email sangitaajmani@gmail.com

Received: August 04, 2017| Published: April 03, 2018

\section{Introduction}

Vitamin D is a fat-soluble vitamin which comes primarily from exposure to sunlight, and is found naturally only in a few foods, such as fish-liver oils, fatty fish, mushrooms, egg yolks, and liver. ${ }^{1}$

Vitamin D status is affected by factors that regulate its production in the skin (i.e. skin pigmentation, latitude, dressing codes, season, aging, sunscreen use, and air pollution) and by factors affecting its absorption or metabolism. ${ }^{1}$

Vitamin D deficiency during pregnancy is associated with impaired maternal musculoskeletal preservation and fetal skeletal formation .Vitamin D deficiency is associated with multiple adverse health outcomes in pregnant female like pre-eclampsia, gestational diabetes mellitus, miscarriage, preterm labor, higher incidence of cesarean section. ${ }^{2-5}$ vitamin D deficiency in pregnancy has important consequences in newborn, including small for gestational age infant, neonatal rickets, tetany, infantile rickets. ${ }^{6-11}$

Many studies have investigated vitamin D deficiency in pregnancy in different ethnic groups and the various methods of supplementation. At present, vitamin D supplementation is not a part of antenatal care programs in India. There is paucity of studies on vitamin D supplementation in pregnant women in India, and its effect on fetomaternal outcome.

\section{Materials and methods}

This study was conducted in the Department of Obstetrics and Gynecology at Kasturba Hospital, Delhi during January 2015-December 2015. The study was conducted after ethical clearance from the institutional ethical committee. The study subjects were recruited from patients attending antenatal clinic of Kasturba hospital.

A written informed consent was taken from all the recruited patients. Women with the following criteria were excluded from the study:

a. Non burka clad pregnant women,

b. Age $<18 \mathrm{yr}$ and $>40 \mathrm{yr}$,

c. Gestational age $<12$ weeks,

d. Subjects residing in place other than Delhi

e. Subjects having liver disease, renal disease, renal calculi, secondary osteoporosis, rheumatoid arthritis, morbid obesity, treatment with antitubercular treatment (ATT) or anti-epileptic in previous 6 months, taking vitamin D or calcium supplementation in previous 1 year.

f. With pre-existing calcium and parathyroid condition, required chronic diuretics or cardiac medication, having chronic 
hypertension (HTN), active thyroid disease. 200 pregnant burka clad pregnant women of 12-16 weeks gestation were included in the study.

They were randomly assigned into two groups that is study group and control group. Study group comprised of 100 pregnant burka clad women of 12-16 weeks gestation who received vitamin D supplementation in the form of oral cholecalciferol sachet $60,000 \mathrm{IU}$ in 5th, 6th and 7th month of gestation along with routine iron, folic acid and calcium supplementation. Control group comprised of 100 pregnant burka clad women of 12-16 weeks who received only routine iron, folic acid and calcium supplementation. At first antenatal visit a detailed history was obtained and thorough examination and routine antenatal investigations of all the subjects was done. At term pregnancy vitamin D level was estimated in both groups and patients were then grouped in three categories: - vitamin D deficient- $<20 \mathrm{ng} /$ $\mathrm{ml}$, vitamin D insufficient $20-30 \mathrm{ng} / \mathrm{ml}$, vitamin D sufficient $>30 \mathrm{ng} /$ $\mathrm{ml}$ Role of vitamin D supplementation and maternal outcome in terms of preeclampsia, gestational diabetes mellitus (GDM), preterm labour, mode of delivery and fetal outcome in terms of low birth weight(LBW),neonatal intensive care unit(NICU) admission, tetany of the two groups was compared.

\section{Statistical evaluation}

All the data was entered in Microsoft excel sheet and various variables were compared by applying chi-square test as a test of significance for nominal and numerical variables with the help of computer using Statistical Package of Social Sciences and Problem Solutions (SPSS)-16.0 software. Statistical significance was considered if $\mathrm{p}$ value was $<0.05$.

\section{Results}

The mean age in this study was 24.6years.Out of 200 patients, $67(33.5 \%)$ patients were primigravida and $133(66.5 \%)$ patients were multigravida. Most of the patients $(66.5 \%)$ belonged to lower socioeconomic class. According to our study vitamin D deficiency was more common in lower socioeconomic status and lower educated. In our study there was no correlation between fish intake and milk, dairy product intake and skin colour with vitamin D deficiency. In our study there is a statistically significant correlation established between vitamin D deficiency and limited outdoor activity and use of sunscreen lotion. Vitamin D supplementation during pregnancy was associated with increased circulating $25(\mathrm{OH})$ vitamin D levels at term. Vitamin D supplementation reduces the incidence of pre-eclampsia and low birth weight neonates. Incidence of gestational diabetes mellitus (GDM), preterm birth, cesarean section, neonatal intensive care unit (NICU) admission, tetany in newborn was not influenced by vitamin D supplementation.

\section{Discussion}

\section{Vitamin D level in vitamin d supplemented and non supplemented group}

$\mathrm{CKH} \mathrm{Yu}$ conducted a prospective randomised study to determine vitamin D status in pregnancy and to evaluate the effects of vitamin D supplementation. A total of 180 women were recruited and randomised into three groups, a single dose of 200,000IU vitamin D, a daily supplement of $800 \mathrm{IU}$ vitamin D from 27 weeks until delivery and a no treatment group. The final maternal vitamin D levels were significantly higher in supplemented group. The result of our study is shown in Table 1 which are similar to above mentioned study (Figure $1)$.
Table I Vitamin D level in vitamin d supplemented and non supplemented group

\begin{tabular}{llllllll}
\hline $\begin{array}{l}\text { Vitamin D } \\
\text { level at term }\end{array}$ & $\begin{array}{l}\text { Group } \\
\text { Vitamin D non }\end{array}$ & $\begin{array}{l}\text { Vitamin D } \\
\text { supplemented }\end{array}$ & Total & P value \\
\hline & NO. $\%$ & NO. & $\%$ & NO. $\%$ & \\
I.Deficient & 83 & $83 \%$ & 2 & $2 \%$ & 85 & $42.50 \%$ & \\
2.Insufficient & 7 & $7 \%$ & 26 & $26 \%$ & 33 & $16.50 \%$ & 0.0005 \\
3.Sufficient & 10 & $10 \%$ & 72 & $72 \%$ & 82 & $41 \%$ & \\
Total & 100 & $100 \%$ & 100 & $100 \%$ & 200 & $100 \%$ & \\
\hline
\end{tabular}

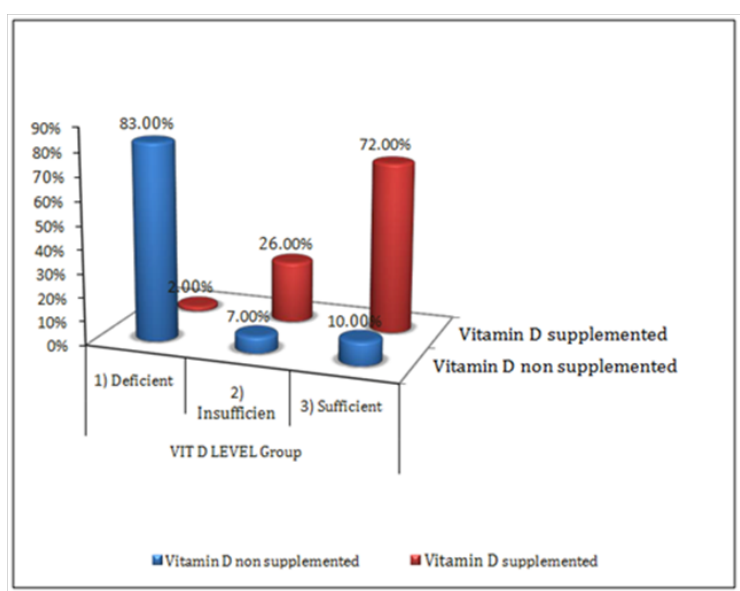

Figure I Vitamin D level in vitamin d supplemented and non supplemented group.

\section{Maternal outcome in vitamin d supplemented and non supplemented group}

Pre-eclampsia: In our study $6 \%$ patients developed pre-eclampsia and out of these $5 \%$ belonged to vitamin D non-supplemented group and $1 \%$ belonged to vitamin $\mathrm{D}$ supplemented making this correlation statistically significant $(p$-value $=0.033)$. These findings suggest that incidence of pre-eclampsia was less in vitamin D supplemented group. Similarly, Bodnar et al. ${ }^{10}$ conducted a case control study of pregnant women in which they found that serum 25-hydroxy vitamin D concentration in early pregnancy were lower in women who subsequently developed preeclampsia compared with controls. Baker also concluded in their study that maternal mid gestation vitamin $\mathrm{D}$ deficiency was associated with increased risk of severe preeclampsia and vitamin D may be a modifiable risk factor for severe preeclampsia. Both of these studies show similar results to our study.

- Gestational diabetes mellitus: In present study $8.5 \%$ patients developed gestational diabetes mellitus. Out of these $5 \%$ belonged to vitamin D non-supplemented group and 3.5\% belonged to vitamin $\mathrm{D}$ supplemented group. The $\mathrm{p}$ - value was 0.447 making this correlation statistically insignificant. Similar results were found by Faustino who conducted a systematic review and metaanalyses of randomised controlled trials to assess the effects of vitamin D supplementation on obstetric outcomes and birth variables and they found that incidence of gestational diabetes mellitus was not influenced by vitamin D supplementation.

- Preterm labour: In our study $7.5 \%$ patients had preterm labour and out of these $4.5 \%$ belonged to vitamin D supplemented group and 3\% belonged to vitamin D non supplemented group. The p-value was 0.421 making this correlation statistically insignificant. Similar results were found in studies conducted by 
Faustino RP and Bodnar LM in both of these studies incidence of preterm birth was not influenced by vitamin $\mathrm{D}$ supplementation.

Mode of delivery: In our study $85 \%$ patients delivered by vaginal route and $15 \%$ patients delivered by LSCS. Out of $15 \%$ patients who delivered by LSCS, $8 \%$ belonged to vitamin D non supplemented group and $7 \%$ belonged to vitamin D supplemented group suggesting no correlation between vitamin D supplementation and mode of delivery (p-value 0.692). Similar result was found by Skowronskajozwaik who recruited 102 pregnant women to study vitamin D3 concentration and its influence on pregnancy course, health of pregnant women and their offspring and found no relation between vitamin D status and incidence of gestational diabetes, mode of delivery and size of newborns (Table 2) (Figure 2).

Table 2 Maternal outcome in vitamin d supplemented and non supplemented group

\begin{tabular}{|c|c|c|c|c|c|c|c|}
\hline \multirow{3}{*}{$\begin{array}{l}\text { Maternal } \\
\text { outcome }\end{array}$} & \multicolumn{7}{|c|}{ Group } \\
\hline & \multicolumn{2}{|c|}{$\begin{array}{l}\text { Vitamin D non } \\
\text { supplemented }\end{array}$} & \multicolumn{2}{|c|}{$\begin{array}{l}\text { Vitamin D } \\
\text { supplemented }\end{array}$} & \multicolumn{2}{|c|}{ Total } & \multirow[t]{2}{*}{ p value } \\
\hline & NO. & $\%$ & NO. & $\%$ & NO. & $\%$ & \\
\hline Pre-Eclampsia & 10 & $10 \%$ & 2 & $2 \%$ & 12 & $6 \%$ & 0.033 \\
\hline $\begin{array}{l}\text { Gestational } \\
\text { Diabetes Mellitus }\end{array}$ & 10 & $10 \%$ & 7 & $7 \%$ & 17 & $8.50 \%$ & 0.447 \\
\hline Preterm Labour & 6 & $6 \%$ & 9 & $9 \%$ & 15 & $7.50 \%$ & 0.421 \\
\hline $\begin{array}{l}\text { Mode of Delivery } \\
\text { (LSCS) }\end{array}$ & 16 & $16 \%$ & 14 & $14 \%$ & 30 & $15 \%$ & 0.692 \\
\hline
\end{tabular}

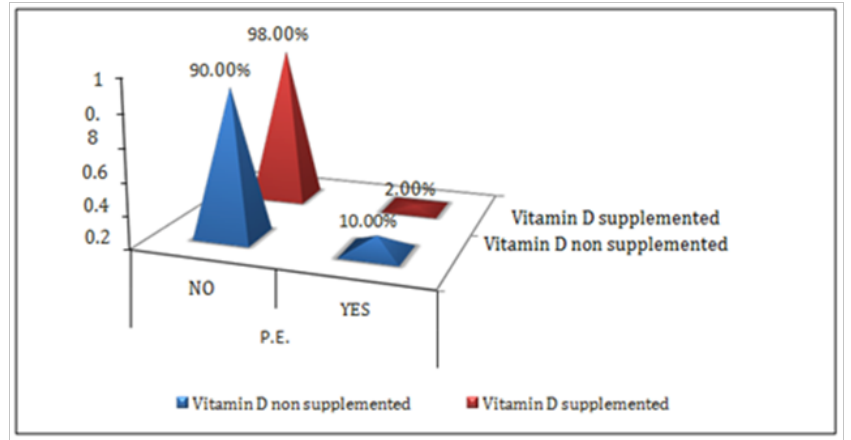

Figure 2 Cases of pre-eclampsia in vitamin $d$ supplemented and non supplemented group.

\section{Fetal outcome in vitamin d supplemented and non} supplemented group

o Low birth weight: In our study, $10 \%$ patients gave birth to LBW neonates $(<2.5 \mathrm{~kg})$. Out of these $7.5 \%$ belonged to vitamin D non supplemented group and $2.5 \%$ belonged to vitamin D supplemented group. The p-value was 0.017 making this correlation statistically significant. Out of 20 patients who gave birth to LBW, $17 \%$ were vitamin D deficient and $2 \%$ had vitamin $\mathrm{D}$ level insufficient and $1 \%$ patient had sufficient level of vitamin D. The p-value was 0.009 making this correlation statistically significant. This implied that vitamin D supplementation could reduce the risk of low birth weight. Similar results were found by Bowyer, De Regil LM.

- Neonatal intensive care unit admission: In our study neonates of $7.5 \%$ patients were admitted in NICU and out of these $5 \%$ mothers belonged to vitamin D non-supplemented group and $2.5 \%$ patients belonged to vitamin $\mathrm{D}$ supplemented group. The p-value was 0.179 making this correlation statistically insignificant. Out of 15 neonates, mothers of $5 \%$ were vitamin
D deficient and $1 \%$ mothers had insufficient level of vitamin D and $1.5 \%$ mothers had sufficient level of vitamin $\mathrm{D}$. The $\mathrm{p}$-value was 0.131 making this correlation statistically insignificant. Hollis studied that there was no significant differences based on supplemented dose in the neonatal intensive care unit admission rate. Although neonatal intensive care unit admission is not a complete assessment measure for neonatal outcome, these findings provide evidence that different levels of vitamin D supplementation had no significant positive or negative effect on the need for advanced neonatal care. The result of this study is comparable with our study.

Neonatal tetany: In our study neonates of 4 patients developed tetany and out of 4 neonates, mothers of 3 belonged to vitamin $\mathrm{D}$ non-supplemented group and 1 patient belonged to vitamin D supplemented group. The p-value was 0.621 making this correlation statistically insignificant. Similarly Cockburn found out that tetanic convulsions occurred in 8 of the 1139 infants $(0.7 \%)$, and there was no difference in the occurrence of covulsions among infants of mothers who received vitamin $\mathrm{D}$, compared to those who did not (Table 3) (Figure 3).

Table 3 Fetal outcome in vitamin d supplemented and non supplemented group

\begin{tabular}{|c|c|c|c|c|c|c|c|}
\hline \multirow{3}{*}{$\begin{array}{l}\text { Fetal } \\
\text { outcome }\end{array}$} & \multicolumn{7}{|c|}{ Group } \\
\hline & \multicolumn{2}{|c|}{$\begin{array}{l}\text { Vitamin D non } \\
\text { supplemented }\end{array}$} & \multicolumn{2}{|c|}{$\begin{array}{l}\text { Vitamin D } \\
\text { Supplemented }\end{array}$} & \multicolumn{2}{|c|}{ Total } & \multirow[t]{2}{*}{$\mathrm{p}$ value } \\
\hline & NO. & Percentage & NO. & Percentage & NO. & Percentage & \\
\hline $\begin{array}{l}\text { Low Birth } \\
\text { Weight }\end{array}$ & 15 & $15 \%$ & 5 & $5 \%$ & 20 & $10 \%$ & 0.017 \\
\hline $\begin{array}{l}\text { Neonatal } \\
\text { Intensive } \\
\text { Care Unit } \\
\text { Admission }\end{array}$ & 10 & $10 \%$ & 5 & $5 \%$ & 15 & $7.50 \%$ & 0.179 \\
\hline Tetany & 3 & $3 \%$ & I & $1 \%$ & 4 & $2 \%$ & 0.621 \\
\hline
\end{tabular}

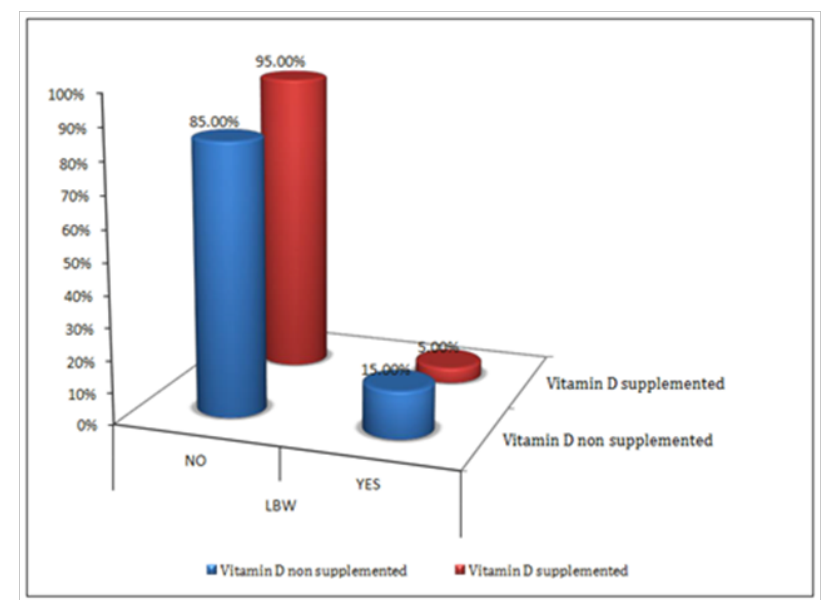

Figure 3 Cases of low birth weight in vitamin $d$ supplemented and non supplemented group.

\section{Conclusion}

Vitamin D deficiency and insufficiency are common in burka clad pregnant women of Delhi. In the present study a significant association was found between vitamin D deficiency and lower socioeconomic status, lower education, limited outdoor activity and with sunscreen lotion use. Vitamin D supplementation during pregnancy was 
associated with increased circulating 25 hydroxy vitamin D levels at term. Vitamin D supplementation reduces the incidence of preeclampsia and low birth weight neonates, hence emphasizing the role of vitamin D supplementation in improving fetomaternal outcome. Incidence of gestational diabetes mellitus, preterm birth, cesarean section, neonatal intensive care unit admission, tetany in newborn was not influenced by vitamin D supplementation in our study.

At present, vitamin D supplementation is not a part of antenatal care programs in India. The US National Academy of Sciences mentions 400IU/day as the reference dietary intake during pregnancy but several investigators worldwide are arguing for revised guidelines for higher vitamin D allowance during pregnancy. This may call for vitamin D supplementation to mothers. Low vitamin D concentrations have been associated with a wide range of adverse maternal and offspring health outcomes. At this time there is insufficient evidence to support a recommendation for screening all pregnant women for vitamin D deficiency. While there is some evidence that vitamin D supplementation could reduce the risk of pre-eclampsia and low birth weight neonates. For pregnant women thought to be at increased risk of vitamin D deficiency, maternal serum 25-hydroxy vitamin D levels can be considered and should be interpreted in the context of the individual clinical circumstance. For recommendations concerning routine vitamin D supplementation during pregnancy, further clinical trials are required with larger sample size.

\section{Acknowledgements}

An informed consent was taken from all the patients recruited in this study.

\section{Conflicts of interest}

There was no conflict of interest among the authors of this study.

\section{Ethics approval and consent to participate}

This study was approved by the institutional ethical committee.

\section{References}

1. Holick MF, Chen TC, Lu Z, et al. Vitamin D and skin physiology: a D-lightful story. J Bone Miner Res. 2007;22(Suppl 2):V28-V33.

2. Holick MF, Binkley NC, Bischoff-Ferrari HA, et al. Evaluation, treatment, and prevention of vitamin D deficiency: an Endocrine Society clinical practice guideline. J Clin Endocrinol Metab. 2011;96(7):1911-30.

3. Aghajafari F, Nagulesapillai T, Ronksley PE, et al. Association between maternal serum 25-hydroxyvitamin D level and pregnancy and neonatal outcomes: systematic review and meta-analysis of observational studies. BMJ. 2013;346:f1169.

4. Bodnar LM, Catov JM, Simhan HN, et al. Maternal vitamin D deficiency increases the risk of pre eclampsia. J Clin Endocrinol Metab. 2007;92(9):3517-3522.

5. Bondar LM, Krohn MA, Simhan HN. Maternal vitamin D deficiency is associated with bacterial vaginosis in the first trimester of pregnancy. $J$ Nutr. 2011;139(6):1157-1161.

6. Zhang CL, Qiu C, Hu FB, et al. Maternal plasma vitamin D concentration and the risk for gestation diabetes mellitus. PLoS One. 2008;3(11) Le3753.

7. Greer FR. 25 hydroxyvitamin D: functional outcomes in infants and young children. Am J Clin Nutr. 2008;88(2):529S-533S.

8. Dawodu A, Wagner CL. Mother-child vitamin D deficiency: an international perspective. Arch Dis Child. 2007;92(9):737-740.

9. Wagner CL, Greer FR. Prevention of Rickets and Vitamin D Deficiency in Infants. Children, and Adolescents. Pediatrics. 2008;122(5):1142-1152.

10. Bodnar LM, Catov JM, Joseph M. Maternal Serum 25-Hydroxyvitamin D concentrations are associated with Small-for-Gestational Age Births in White Women. J Nutr. 2010;140(5):999-1006.

11. Catov JM, Bodnar LM, Olsen JJ, et al. Periconceptional multivitamin use and risk of preterm or small-for-gestational-age births in the Danish National Birth Cohort. Am J Clin Nutr. 2011;94(3):906-912. 